tween calculated and observed frequencies, it is evident that their assignment of numerical frequency values is incorrect where it differs significantly from that of Miller and Inskeep.

Further confirmation of Kilpatrick, Pitzer, and Spitzer's concept of pseudorotation has been found in investigations of thiacyclopentane, ${ }^{5}$ a restricted pseudorotator, and pyrrolidine, ${ }^{6}$ a free (or only slightly restricted) pseudorotator. The consistent treatment found necessary for these two substances and for cyclopentane is conclusive evidence that such molecules do undergo pseudorotation as originally proposed by Kilpatrick et al.

* Contribution No. 76 from the Thermodynamics Laboratory Petroleum Experiment Station, Bureau of Mines, United States Department of the Interior.

${ }_{1}$ Kilpatrick, Pitzer, and Spitzer, J. Am. Chem. Soc. 69, 2483 (1947).

${ }^{2}$ F. A. Miller and R. G. Inskeep, J. Chem. Phys. 18, 1519 (1950).

3 B. Curnutte and W. H. Shaffer, J. Mol. Spectroscopy 1, 239 (1957).

${ }^{4}$ R. Spitzer and K. S. Pitzer, J. Am. Chem. Soc. 68, 2537 (1946).

${ }^{5}$ W. N. Hubbard et al., J. Am. Chem. Soc. 74, 6025 (1952).

${ }^{6}$ Unpublished results, Thermodynamics Laboratory, Bureau of Mines, Bartlesville, Oklahoma.

\section{Suction of the Boundary-Layer Flow along a Hot Plate as a Means of Separating Gas Mixtures*}

\section{C. LIU}

Engineering Research Institute, The University of Michigan, Ann Arbor, Michigan

(Received June 9, 1958)

W HEN a mixture of gases flows along a hot plate, a thin layer of laminar shear flow with extremely high-temperature gradient- - called thermal boundary layer-appears. The thermal gradient across the boundary layer depends upon the difference of temperatures of the plate and the free-stream gases, the flow and gas parameters, ${ }^{1}$ e.g., Reynolds number, Mach number, and Prandtl number $\left(\sigma=\mu c_{p} / \lambda\right)$. If the mixture consists of gases of unequal molecular weights, thermal-diffusion flux will be set up so that the lighter gas tends to move to the hot region and the heavier gas, to the cold region. When the temperature of the plate is maintained much higher than that of the free stream, the concentration of the lighter gas increases monotonically from its free-stream value at the outer edge of the boundary layer to a maximum at the plate. Suction of the mixture at the plate, through either slots or porous wall, serves two purposes: (1) collection of the enriched light mixture, and (2) rendering the boundary layer more favorable for diffusive separation by boosting its temperature gradient and delaying its transition to the turbulent state.

In the following, a summary of the theory of diffusive separation in the boundary layer along a hot plate with surface suction is given. In the analysis, a flat plate with constant suction is assumed. It is also assumed that the concentration of the lighter gas is much smaller than unity. Notations used in reference 2 are followed, except that $\theta$ substitutes for $n_{10}, D$ for $D_{12}, V(u, v)$ for $c_{0}\left(u_{0}, v_{0}\right)$, and $m_{2}>m_{1}$. Note that the pressure gradient across the boundary layer is negligible. From the application of the equation of continuity to the total flow and to the light component flow with thermaldiffusion flux, ${ }^{2}$ we obtain the equation of concentration $(\theta)$ distribution:

$$
\begin{aligned}
n(\partial \theta / \partial t)+n V \cdot \nabla \theta- & {[(\beta-\theta) / \beta] \nabla \cdot } \\
\cdot & \{n D[\nabla \theta-\alpha \theta(1-\theta) \nabla \ln T]\}=0,
\end{aligned}
$$

where $\beta=m_{2} /\left(m_{2}-m_{1}\right)$. It is assumed that $n D /(n D)_{\infty}=$ $\left(T / T_{\infty}\right)^{s}$, where $s$ is constant for a given mixture, and subscript $\infty$ denotes the condition at the free-stream edge of the boundary layer. By applying the order of magnitude analysis used in usual boundary-layer approximations, namely, $u \gg v$ and $\partial / \partial y \gg \partial / \partial x$, we can simplify Eq. (1), for the case $\theta \ll 1$, into

$$
\begin{aligned}
n(\partial \theta / \partial t)+ & n u(\partial \theta / \partial x)+n v(\partial \theta / \partial y) \\
& =\partial / \partial y\{n D[\partial \theta / \partial y)-\alpha \theta(\partial \ln T / \partial y)]\} .
\end{aligned}
$$

In the case of flow along a flat plate with constant suction, both the velocity and temperature distribution in the boundary layer approach their respective asymptotic profiles in a short distance $e^{1}$ from the leading edge; the asymptotic profile is given by ${ }^{3}$

$T / T_{\infty}=1-\left(1-T_{0} / T_{\infty}\right) e^{-\sigma \zeta}$

$$
+\frac{1}{2}(\gamma-1) M_{\infty}^{2} \sigma\left(e^{-\sigma \zeta}-e^{-2 \zeta}\right) /(2-\sigma),
$$

where $\sigma=\mu c_{p} / \lambda$ is assumed constant and

$$
\zeta=-\rho_{0} v_{0} \int_{0}^{y} d y / \mu
$$

Subscript 0 denotes the condition at the plate; $M_{\infty}$ is the free-stream Mach number.

It can also be shown that the relaxation time for $\theta$ to reach equilibrium is small; we need to solve Eq. (2) only for its steady state, which becomes

$$
\theta^{\prime \prime}+S \theta^{\prime}-\alpha\left[\theta(\ln T)^{\prime}\right]^{\prime}=0
$$

where the prime denotes $d / d \zeta ; S=\mu / \rho D$. The solution to Eq. (4) together with the boundary conditions $\theta=\theta_{\infty}$ and $\theta^{\prime}=0$ at $\zeta=\infty$, in the case $M_{\infty} \ll 1$, leads to the enrichment factor

$$
\begin{aligned}
& \left(\theta_{0}-\theta_{\infty}\right) / \theta_{\infty}=1-\alpha\left(1-T_{0} / T_{\infty}\right)\left(T_{0} / T_{\infty}\right)^{\alpha} \\
& \cdot(1-\Lambda)^{-1} F\left(1+\alpha, 1-\Lambda ; 2-\Lambda ; 1-T_{0} / T_{\infty}\right),
\end{aligned}
$$

where $F$ denotes the hypergeometric function ${ }^{4} ; \Lambda=S / \sigma$ lies between 0 and 1 .

It is well known ${ }^{5}$ that the enrichment factor for Clusius and Dickel's thermal-diffusion column is $T_{0}{ }^{\alpha} / T_{\infty}{ }^{\alpha}-1$. The enrichment factor as obtained from 
Eq. (5), for isotopes with $0.01<\alpha<0.1$ and $T_{0} / T_{\infty}=2$, is about 14 times greater. The possible contribution to mixture separation by capillary effect ${ }^{6}$ through the porous wall is not included. The surprising effectiveness of mixture separation by suction of the thermaldiffusion boundary layer is attributed to the unusually high temperature gradient existing in the boundary layer near the plate. It should be noted that the present method cannot compare with the diffusion-column method from the standpoint of economy or power consumed in separating a mixture.

An experimental program is being planned.

* The present work is supported by the Meteorological Branch of Evans Signal Laboratory (Contract No. DA-36-039 SC-64659). Stimulating discussions with Professor Sydney Chapman are gratefully acknowledged.

${ }_{1}^{1} \mathrm{H}$. Schlichting, Boundary Layer Theory (McGraw-Hill Book Company, Inc., New York, 1955)

${ }^{2} \mathrm{~S}$. Chapman and T. G. Cowling, The Mathematical Theory of Nonuniform Gases (Cambridge University Press, New York, 1952).

${ }^{3}$ L. Howarth, editor, Modern Development in Fluid Dynamics, High Speed Flow (Oxford University Press, New York, 1953), Vol. I.

${ }^{4} \mathrm{~W}$. Magnus and F. Oberhettinger, Formulas and Theorems for the Functions of Mathematical Physics (Chelsea Publishing Company, New York, 1954).

R. C. Clark and W. H. Furry, Revs. Modern Phys. 18, 151 (1956).

${ }^{6}$ R. D. Present and A. J. Debethune, Phys. Rev. 75, 1050 (1949).

\section{Mass Spectrometric Observation of Triatomic Ions in Chlorine and Bromine Gases}

\author{
C. E. Melton, Gus A. Ropp, and P. S. Rudolph \\ Chemistry Division, Oak Ridge National Laboratory, ${ }^{*}$ \\ Oak Ridge, Tennessee
}

(Received December 19, 1957)

$\mathbf{P}$ RELUSIVE to using the mass spectrometer in the study of primary products (ions, radicals, and/or molecules) formed during the radiolysis of gases, mass spectrometric studies ${ }^{1}$ of the photochemical reaction of chlorine with formic $\operatorname{acid}^{2,3}$ were made. In these studies the ions $\mathrm{Cl}_{3}{ }^{+}$and $\mathrm{Cl}_{3}^{-}$were observed. These species, which have not been previously reported, were also detected in pure chlorine. Rollefson and Eyring ${ }^{4}$ have predicted the stability of neutral $\mathrm{Cl}_{3}$. This molecule has also been suggested as the active intermediate in a number of proposed mechanisms of photochemical reactions of chlorine. We were interested, therefore, in learning whether the observed triatomic chlorine ions resulted from ionization of neutral $\mathrm{Cl}_{3}$.

Data were obtained using an especially constructed six-inch radius, $60^{\circ}$ sector type mass spectrometer. The detection system consisted of a fourteen stage electron multiplier with a gain of $10^{8}$, a vibrating reed electrometer, and a counting circuit. Currents as small as 5 ions per second could be detected. However, the minimum useable signal was considered to be 20 ions per sec or $3.2 \times 10^{-18} \mathrm{amp}$. Since the customary tungsten filament used to produce ionizing electrons was attacked by chlorine, it was replaced by a relatively inert thoria-iridium filament. ${ }^{5}$ Further details of equipment, calibration, and instrument performance are given elsewhere. ${ }^{6,7}$

Typical scans of the spectra of the triatomic chlorine ions are shown in Fig. 1. Experimental values from ten

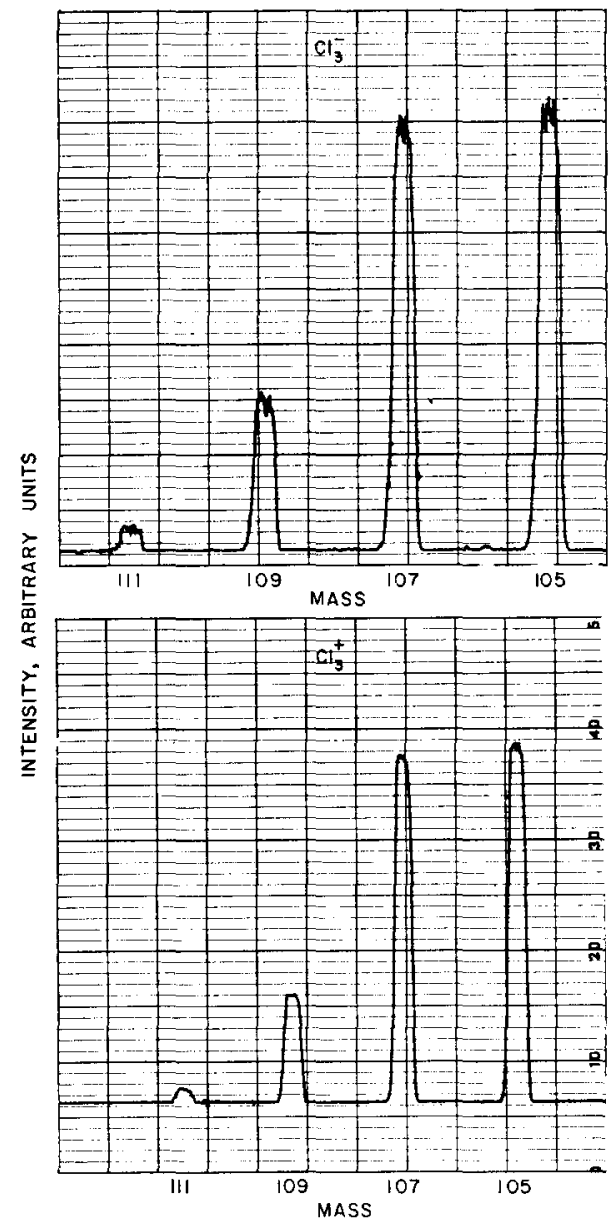

FIG. 1. Typical scans of the spectra of the triatomic chlorine ions.

separate determinations of the relative isotopic abundances agree with the calculated values within an experimental error of about $\pm 0.2 \%$. These calculations were based on the adopted ${ }^{8}$ value of $75.4 \% \mathrm{Cl}^{35}$ assuming negligible isotopic discrimination. ${ }^{9}$ The relative abundance of $\mathrm{Cl}_{3}{ }^{+}$normalized to $\mathrm{Cl}_{2}{ }^{+}=100$ varied from $0.02 \%$ to $0.1 \%$ over the pressure range of $1 \times 10^{-5}$ $\mathrm{mm}$ to $1 \times 10^{-4} \mathrm{~mm}$. A similar scan of the $\mathrm{Br}_{3}{ }^{+}$spectrum of bromine vapor is shown in Fig. 2. The negative species $\mathrm{Br}_{3}{ }^{+}$spectrum of bromine vapor is shown in Fig. 2. The negative species $\mathrm{Br}_{3}{ }^{-}$was also detected.

An extensive investigation failed to establish whether any of the trimeric chlorine ions were derived from 